\title{
Two models for linear comparative calibration
}

\author{
G. Wimmer ${ }^{1,2,3, \star}$ and V. Witkovský ${ }^{4}$ \\ 1 Mathematical Institute, Slovak Academy of Sciences Bratislava, Slovak Republic \\ 2 Faculty of Natural Sciences, Matej Bel University Banská Bystrica, Slovak Republic \\ 3 Institute of Mathematics and Statistics, Masaryk University Brno, Czech Republic \\ 4 Institute of Measurement Science, Slovak Academy of Sciences Bratislava, Slovak Republic
}

Received: 10 October 2012 / Accepted: 28 October 2012

\begin{abstract}
We consider the comparative calibration problem in the case when linear relationship is assumed between two considered measuring devices with possibly different units and precisions. The first method for obtaining the approximate confidence region for unknown parameters of the calibration line applies the maximum likelihood estimators of the unknown parameters. The second method is based on estimation of the calibration line via replicated errors-in-variables model. Essential point in this approach is approximation of the small sample distribution of the Wald-type test statistic. This enables to construct the interval estimators for the multiple-use calibration case.
\end{abstract}

Keywords: Calibration problem; multiple-use calibration; maximum likelihood estimator; errors-invariables model; Kenward-Roger approximation

\section{Introduction}

We consider the problem of comparative calibration in small sample case. This paper presents two methods for obtaining the approximate confidence region for unknown parameters of the calibration line and to construct the interval estimators for the multiple-use calibration case. This is useful for linear univariate comparative calibration problem with possibly different and unknown precisions of both measuring devices and enables to construct the interval estimators for the unknown quantity in multipleuse calibration case.

The first method for obtaining the approximate confidence region for unknown parameters of the calibration line applies the maximum likelihood estimators (MLE) of the unknown parameters and relies on the asymptotic properties of the MLEs which are used for construction of the Scheffé-type confidence region for the calibration line. From statistical point of view, the second method is based on the linear errors-in-variables (EIV) model. In a standard situation, the estimators of the calibration function parameters are based on minimization of the weighted total sum of squares in the orthogonal regression with weights inversely proportional to the true standard deviations. If the true standard deviations are (partially or completely) unknown, and should be estimated from the measurements, we suggest to use an alternative iterative algorithm based on locally linearized model for parameter estimation that allows to consider the problem of deriving the approximate confidence region for the parameters. The

^ Correspondence: wimmer@mat.savba.sk confidence limits are derived using the concept of Kenward and Roger, see [1] and also [2-5].

The suggested estimation methods enables to construct the interval estimators for the multiple-use calibration case. In this paper we present and illustrate both methods for construction the approximate confidence interval for the true value of the measurand (in units of the more precise device) in the multiple-use calibration case.

\section{Methods}

\subsection{Estimation of the calibration line parameters via maximum likelihood method}

Let us have measurements $X_{11}, Y_{11}, \ldots, X_{n 1}, Y_{n 1}$. We suppose that the measurements are normally distributed, independent and it is valid that the mean value of $Y_{i 1}$ is

$$
\mathcal{E}\left(Y_{i 1}\right)=a+b \mu_{i}, \quad i=1,2, \ldots, n,
$$

where $\mu_{i}$ is the mean value of $X_{i 1}$ and $a, b$ are the unknown parameters of the calibration line (more see in [6]). We consider this group of $2 n$ measurements as one (single) experiment. The likelihood function of the random vector 


$$
\begin{aligned}
& \left(X_{11}, Y_{11}, \ldots, X_{n 1}, Y_{n 1}\right)^{\prime} \text { is } \\
& \begin{aligned}
L\left(a, b, \sigma_{x}^{2}, \sigma_{y}^{2}, \mu_{1}, \ldots, \mu_{n} \mid x_{11}, y_{11}, \ldots, x_{n 1}, y_{n 1}\right) \\
=\prod_{i=1}^{n} f_{i}\left(x_{i 1} ; \mu_{i}, \sigma_{x}^{2}\right) \prod_{j=1}^{n} g_{j}\left(y_{j 1} ; a+b \mu_{j}, \sigma_{y}^{2}\right) \\
=\frac{1}{(2 \pi)^{n} \sigma_{x}^{n} \sigma_{y}^{n}} \exp \left\{-\frac{1}{2 \sigma_{x}^{2}} \sum_{i=1}^{n}\left(x_{i 1}-\mu_{i}\right)^{2}\right. \\
\left.\quad-\frac{1}{2 \sigma_{y}^{2}} \sum_{j=1}^{n}\left(y_{j 1}-a-b \mu_{j}\right)^{2}\right\} .
\end{aligned}
\end{aligned}
$$

We shall assume that this experiment is repeated independently $m$-times. The $r$-th experiment is modeled by the random vector $\left(X_{1 r}, Y_{1 r}, \ldots, X_{n r}, Y_{n r}\right)^{\prime}$. The likelihood function of the whole calibration experiment (consisting of $m$ experiments) is

$$
\begin{aligned}
& L\left(a, b, \sigma_{x}^{2}, \sigma_{y}^{2}, \mu_{1}, \ldots, \mu_{n} \mid x_{11}, y_{11}, \ldots, x_{n m}, y_{n m}\right) \\
& =\frac{1}{(2 \pi)^{n m} \sigma_{x}^{n m} \sigma_{y}^{n m}} \exp \left\{-\frac{1}{2 \sigma_{x}^{2}} \sum_{i=1}^{n} \sum_{j=1}^{m}\left(x_{i j}-\mu_{i}\right)^{2}\right. \\
& \left.-\frac{1}{2 \sigma_{y}^{2}} \sum_{i=1}^{n} \sum_{j=1}^{m}\left(y_{i j}-a-b \mu_{i}\right)^{2}\right\} .
\end{aligned}
$$

The maximum likelihood estimators $\tilde{a}\left(X_{11}, \ldots, Y_{n m}\right)$, $\tilde{b}\left(X_{11}, \ldots, Y_{n m}\right), \quad \tilde{\sigma}_{x}^{2}\left(X_{11}, \ldots, Y_{n m}\right), \quad \tilde{\sigma}_{y}^{2}\left(X_{11}, \ldots, Y_{n m}\right)$, $\tilde{\mu}_{1}\left(X_{11}, \ldots, Y_{n m}\right), \ldots, \tilde{\mu}_{n}\left(X_{11}, \ldots, Y_{n m}\right)$ are solutions of the likelihood equations

$$
\begin{gathered}
\sum_{i=1}^{n} \sum_{j=1}^{m}\left(Y_{i j}-\tilde{a}-\tilde{b} \tilde{\mu}_{i}\right)=0 \\
\sum_{i=1}^{n} \sum_{j=1}^{m}\left(Y_{i j}-\tilde{a}-\tilde{b} \tilde{\mu}_{i}\right) \tilde{\mu}_{i}=0 \\
\sum_{i=1}^{n} \sum_{j=1}^{m}\left(X_{i j}-\tilde{\mu}_{i}\right)^{2}=m n \tilde{\sigma}_{x}^{2}, \\
\sum_{i=1}^{n} \sum_{j=1}^{m}\left(Y_{i j}-\tilde{a}-\tilde{b} \tilde{\mu}_{i}\right)^{2}=m n \tilde{\sigma}_{y}^{2}, \\
\tilde{\sigma}_{y}^{2} \sum_{j=1}^{m}\left(X_{1 j}-\tilde{\mu}_{1}\right)=-\tilde{\sigma}_{x}^{2} \tilde{b} \sum_{j=1}^{m}\left(Y_{1 j}-\tilde{a}-\tilde{b} \tilde{\mu}_{1}\right) \\
\vdots \\
\tilde{\sigma}_{y}^{2} \sum_{j=1}^{m}\left(X_{n j}-\tilde{\mu}_{n}\right)=-\tilde{\sigma}_{x}^{2} \tilde{b} \sum_{j=1}^{m}\left(Y_{n j}-\tilde{a}-\tilde{b} \tilde{\mu}_{n}\right) .
\end{gathered}
$$

It is valid

$$
\sqrt{m}\left(\begin{array}{c}
\tilde{a}-a \\
\tilde{b}-b
\end{array}\right) \stackrel{\mathcal{D}}{\longrightarrow} N\left(\left(\begin{array}{l}
0 \\
0
\end{array}\right), \boldsymbol{\Sigma}\right)
$$

(convergence in distribution). The asymptotic covariance matrix of the likelidood estimator $(\tilde{a}, \tilde{b})^{\prime}$ is

$$
\begin{aligned}
\boldsymbol{\Sigma}= & \frac{b^{2} \sigma_{x}^{2}+\sigma_{y}^{2}}{m\left(n \sum_{i=1}^{n} \mu_{i}^{2}-\left(\sum_{j=1}^{n} \mu_{j}\right)^{2}\right)} \\
& \times\left(\begin{array}{cc}
\sum_{i=1}^{n} \mu_{i}^{2} & -\sum_{i=1}^{n} \mu_{i} \\
-\sum_{i=1}^{n} \mu_{i} & n
\end{array}\right) .
\end{aligned}
$$

We use the approximative $\tilde{\boldsymbol{\Sigma}}$

$$
\begin{aligned}
\tilde{\boldsymbol{\Sigma}}= & \frac{\tilde{b}^{2} \tilde{\sigma}_{x}^{2}+\tilde{\sigma}_{y}^{2}}{m\left(n \sum_{i=1}^{n} \tilde{\mu}_{i}^{2}-\left(\sum_{j=1}^{n} \tilde{\mu}_{j}\right)^{2}\right)} \\
& \times\left(\begin{array}{cc}
\sum_{i=1}^{n} \tilde{\mu}_{i}^{2} & -\sum_{i=1}^{n} \tilde{\mu}_{i} \\
-\sum_{i=1}^{n} \tilde{\mu}_{i} & n
\end{array}\right) .
\end{aligned}
$$

Further, it holds

$$
\chi^{2}=\left(\begin{array}{c}
\tilde{a}-a \\
\tilde{b}-b
\end{array}\right)^{\prime} \tilde{\Sigma}^{-1}\left(\begin{array}{c}
\tilde{a}-a \\
\tilde{b}-b
\end{array}\right) \stackrel{\text { approx }}{\sim} \chi_{2}^{2},
$$

and

$$
\operatorname{Pr}\left\{\left(\begin{array}{c}
\tilde{a}-a \\
\tilde{b}-b
\end{array}\right)^{\prime} \tilde{\boldsymbol{\Sigma}}^{-1}\left(\begin{array}{c}
\tilde{a}-a \\
\tilde{b}-b
\end{array}\right) \leqq \chi_{2}^{2}(1-\alpha)\right\} \stackrel{\text { approx. }}{=} 1-\alpha,
$$

$\left(\chi_{2}^{2}(1-\alpha)\right.$ is the $(1-\alpha)$-quantile of the $\chi^{2}$ distribution with 2 degrees of freedom).

From that we obtain the ML (approximative, asymptotic) $(1-\alpha)$-confidence region for $(a, b)^{\prime}$

$$
\mathcal{C}_{(1-\alpha)}^{M L}=\left\{\left(\begin{array}{l}
a \\
b
\end{array}\right):\left(\begin{array}{l}
\tilde{a}-a \\
\tilde{b}-b
\end{array}\right)^{\prime} \tilde{\Sigma}^{-1}\left(\begin{array}{l}
\tilde{a}-a \\
\tilde{b}-b
\end{array}\right) \leqq \chi_{2}^{2}(1-\alpha)\right\}
$$

\subsection{Estimation of the calibration line parameters via replicated errors-in-variables model}

Calibration experiment we can model using EIV model

$$
Y_{i}=\alpha+\beta \mu_{i}+\varepsilon_{i}, \quad X_{i}=\mu_{i}+\delta_{i}
$$

$\left(\varepsilon_{i} \sim N\left(0, \sigma_{Y}^{2}\right), \delta_{i} \sim N\left(0, \sigma_{X}^{2}\right)\right.$ independent). Written in another way

$$
\mathcal{E}\left(Y_{i}\right)=\alpha+\beta \mu_{i}, \quad \mathcal{E}\left(X_{i}\right)=\mu_{i} .
$$

Vectors of errorless measurements realized using instruments $\mathrm{A}$ and $\mathrm{B}$ are $\boldsymbol{\mu}=\left(\mu_{1}, \mu_{2}, \ldots, \mu_{n}\right)^{\prime}$ and $\boldsymbol{\nu}=$ $\left(\nu_{1}, \nu_{2}, \ldots, \nu_{n}\right)^{\prime}$. Vector of measurements with instrument $\mathrm{A}$ is $\mathbf{X}_{n, 1} \sim N\left(\boldsymbol{\mu} ; \sigma_{x}^{2} \mathbf{I}_{n, n}\right)$. Vector of measurements with instrument $\mathrm{B}$ is $\mathbf{Y}_{n, 1} \sim N\left(\boldsymbol{\nu} ; \sigma_{y}^{2} \mathbf{I}_{n, n}\right)$. We obtain the model

$$
\left(\begin{array}{l}
\mathbf{X} \\
\mathbf{Y}
\end{array}\right) \sim N\left[\left(\begin{array}{l}
\boldsymbol{\mu} \\
\boldsymbol{\nu}
\end{array}\right),\left(\begin{array}{cc}
\sigma_{x}^{2} \mathbf{I} & \mathbf{0} \\
\mathbf{0} & \sigma_{y}^{2} \mathbf{I}
\end{array}\right)\right]
$$

with condition on parameters

$$
\boldsymbol{\nu}=a \mathbf{1}_{n, 1}+b \boldsymbol{\mu},
$$


where $\mathbf{1}_{n, 1}=(1,1, \ldots, 1)^{\prime}$. First we linearize the model using Taylor series in a neighborhood of $\boldsymbol{\mu}_{0}=$ $\left(\mu_{01}, \mu_{02}, \ldots, \mu_{0 n}\right)^{\prime}$ a $b_{0}$ (some values near the reality $\boldsymbol{\mu}$ a $b)$. Now $\boldsymbol{\mu}=\boldsymbol{\mu}_{0}+\delta \boldsymbol{\mu}, b=b_{0}+\delta b$ and the new model parameters are $\delta \boldsymbol{\mu}=\left(\delta \mu_{1}, \delta \mu_{2}, \ldots, \delta \mu_{n}\right)^{\prime}, \boldsymbol{\nu}, a, \delta b, \sigma_{x}^{2}, \sigma_{y}^{2}$. We get the (approximative) linear regression model

$$
\left(\begin{array}{c}
\boldsymbol{X}-\boldsymbol{\mu}_{0} \\
\boldsymbol{Y}
\end{array}\right) \sim N\left[\left(\begin{array}{c}
\delta \boldsymbol{\mu} \\
\boldsymbol{\nu}
\end{array}\right),\left(\begin{array}{cc}
\sigma_{x}^{2} \boldsymbol{I} & \mathbf{0} \\
\mathbf{0} & \sigma_{y}^{2} \boldsymbol{I}
\end{array}\right)\right]
$$

with (linear) conditions on parameters

$$
b_{0} \boldsymbol{\mu}_{0}+\left(b_{0} \boldsymbol{I}:-\boldsymbol{I}\right)\left(\begin{array}{c}
\delta \boldsymbol{\mu} \\
\boldsymbol{\nu}
\end{array}\right)+\left(\mathbf{1}, \boldsymbol{\mu}_{0}\right)\left(\begin{array}{c}
a \\
\delta b
\end{array}\right)=\mathbf{0}
$$

Dispersions $\sigma_{x}^{2}$ and $\sigma_{y}^{2}$ are unknown. One possibility to estimate them are the $\left(\sigma_{x 0}^{2}, \sigma_{y 0}^{2}\right)$-MINQUE estimators (minimum norm quadratic unbiased estimator). As this estimators do not exist in model (1)-(2), we need to repeat the whole experiment $m$ times independently. The repeated measurements are $\boldsymbol{X}_{j}=\left(X_{j 1}, \ldots, X_{j n}\right)^{\prime}, \boldsymbol{Y}_{j}=$ $\left(Y_{j 1}, \ldots, Y_{j n}\right)^{\prime}, j=1, \ldots, m$. The best linear unbiased estimators $\boldsymbol{\mu}, \boldsymbol{\nu}, a, \delta b$ in replicated model are (see [2])

$$
\begin{gathered}
\hat{\boldsymbol{\mu}}=\overline{\boldsymbol{X}}+\frac{b_{0} \sigma_{x}^{2}}{b_{0}^{2} \sigma_{x}^{2}+\sigma_{y}^{2}} \boldsymbol{M}_{\left[1, \mu_{0}\right]}\left(\overline{\boldsymbol{Y}}-b_{0} \overline{\boldsymbol{X}}\right), \\
\hat{\boldsymbol{\nu}}=\overline{\boldsymbol{Y}}-\frac{\sigma_{y}^{2}}{b_{0}^{2} \sigma_{x}^{2}+\sigma_{y}^{2}} \boldsymbol{M}_{\left[1, \mu_{0}\right]}\left(\overline{\boldsymbol{Y}}-b_{0} \overline{\boldsymbol{X}}\right), \\
\left(\begin{array}{c}
\hat{a} \\
\hat{\delta b}
\end{array}\right)=\left(\begin{array}{cc}
n & \mathbf{1}^{\prime} \boldsymbol{\mu}_{0} \\
\boldsymbol{\mu}_{0}^{\prime} \mathbf{1} & \boldsymbol{\mu}_{0}^{\prime} \boldsymbol{\mu}_{0}
\end{array}\right)^{-1}\left(\begin{array}{c}
\mathbf{1}^{\prime}\left(\overline{\boldsymbol{Y}}-b_{0} \overline{\boldsymbol{X}}\right) \\
\boldsymbol{\mu}_{0}^{\prime}\left(\overline{\boldsymbol{Y}}-b_{0} \overline{\boldsymbol{X}}\right)
\end{array}\right),
\end{gathered}
$$

with the covariance matrix

$$
\operatorname{cov}\left(\begin{array}{c}
\hat{a} \\
\hat{b}
\end{array}\right)=\frac{b_{0}^{2} \sigma_{x}^{2}+\sigma_{y}^{2}}{m}\left(\begin{array}{cc}
n & \mathbf{1}^{\prime} \boldsymbol{\mu}_{0} \\
\boldsymbol{\mu}_{0}^{\prime} \mathbf{1} & \boldsymbol{\mu}_{0}^{\prime} \boldsymbol{\mu}_{0}
\end{array}\right)^{-1},
$$

where

$$
\overline{\boldsymbol{X}}=\frac{1}{m} \sum_{j=1}^{m} \boldsymbol{X}_{j}, \quad \overline{\boldsymbol{Y}}=\frac{1}{m} \sum_{j=1}^{m} \boldsymbol{Y}_{j}
$$

and

$$
\boldsymbol{M}_{\left[1, \mu_{0}\right]}=\boldsymbol{I}-\left[\mathbf{1}, \boldsymbol{\mu}_{0}\right]\left(\left[\mathbf{1}, \boldsymbol{\mu}_{0}\right]^{\prime}\left[\mathbf{1}, \boldsymbol{\mu}_{0}\right]\right)^{-1}\left[\mathbf{1}, \boldsymbol{\mu}_{0}\right]^{\prime} .
$$

$\left(\sigma_{x 0}^{2}, \sigma_{y 0}^{2}\right)$-MINQUE estimators of $\sigma_{x}^{2}$ and $\sigma_{y}^{2}$ in replicated model are

$$
\left(\begin{array}{c}
\hat{\sigma}_{x}^{2} \\
\hat{\sigma}_{y}^{2}
\end{array}\right)=\frac{1}{n(m-1)}\left[\boldsymbol{I}_{2,2}-c_{0}\left(\begin{array}{cc}
b_{0}^{4} \sigma_{x 0}^{4} & b_{0}^{2} \sigma_{x 0}^{4} \\
b_{0}^{2} \sigma_{y 0}^{4} & \sigma_{y 0}^{4}
\end{array}\right)\right]\left(\begin{array}{c}
\hat{\kappa}_{1} \\
\hat{\kappa}_{2}
\end{array}\right),
$$

where

$$
\begin{gathered}
c_{0}=\frac{n-2}{\left(b_{0}^{4} \sigma_{x 0}^{4}+\sigma_{y 0}^{4}\right)(m n-2)+2 b_{0}^{2} \sigma_{x 0}^{2} \sigma_{y 0}^{2}(m-1) n}, \\
\hat{\kappa}_{1}=\sum_{j=1}^{m}\left(\boldsymbol{X}_{j}-\overline{\boldsymbol{X}}\right)^{\prime}\left(\boldsymbol{X}_{j}-\overline{\boldsymbol{X}}\right)+m(\overline{\boldsymbol{X}}-\hat{\boldsymbol{\mu}})^{\prime}(\overline{\boldsymbol{X}}-\hat{\boldsymbol{\mu}}),
\end{gathered}
$$

$$
\hat{\kappa}_{2}=\sum_{j=1}^{m}\left(\boldsymbol{Y}_{j}-\overline{\boldsymbol{Y}}\right)^{\prime}\left(\boldsymbol{Y}_{j}-\overline{\boldsymbol{Y}}\right)+m(\overline{\boldsymbol{Y}}-\hat{\boldsymbol{\nu}})^{\prime}(\overline{\boldsymbol{Y}}-\hat{\boldsymbol{\nu}}) .
$$

The covariance matrix of the estimators (6) (local in the values $\left.\left(\sigma_{x 0}^{2}, \sigma_{y 0}^{2}\right)\right)$ is

$$
\begin{aligned}
\boldsymbol{W} & =\left(\begin{array}{ll}
w_{11} & w_{12} \\
w_{21} & w_{22}
\end{array}\right) \\
& =\frac{2}{n(m-1)}\left[\boldsymbol{I}_{2,2}-c_{0}\left(\begin{array}{cc}
b_{0}^{4} \sigma_{x 0}^{4} & b_{0}^{2} \sigma_{x 0}^{4} \\
b_{0}^{2} \sigma_{y 0}^{4} & \sigma_{y 0}^{4}
\end{array}\right)\right]\left(\begin{array}{cc}
\sigma_{x 0}^{4} & 0 \\
0 & \sigma_{y 0}^{4}
\end{array}\right) .
\end{aligned}
$$

A natural choice of the initial values resulting from the measurements can be as follows

$$
\begin{aligned}
\boldsymbol{\mu}_{0} & =\overline{\boldsymbol{X}}, \quad b_{0}=\frac{n \overline{\boldsymbol{X}}^{\prime} \overline{\boldsymbol{Y}}-\left(\mathbf{1}^{\prime} \overline{\boldsymbol{X}}\right)\left(\mathbf{1}^{\prime} \overline{\boldsymbol{Y}}\right)}{n \overline{\boldsymbol{X}}^{\prime} \overline{\boldsymbol{X}}-\left(\mathbf{1}^{\prime} \overline{\boldsymbol{X}}\right)^{2}}, \\
\sigma_{x 0}^{2} & =\frac{1}{n(m-1)} \sum_{i=1}^{n} \sum_{j=1}^{m}\left(X_{j i}-\bar{X}_{i}\right)^{2} \\
\sigma_{y 0}^{2} & =\frac{1}{n(m-1)} \sum_{i=1}^{n} \sum_{j=1}^{m}\left(Y_{j i}-\bar{Y}_{i}\right)^{2} .
\end{aligned}
$$

Further the estimates are computed as follows: $\hat{a}, \hat{b}$ from (5), $\hat{\boldsymbol{\mu}}$ from (3), $\hat{\boldsymbol{\nu}}$ from (4), $\hat{\sigma}_{x}^{2}$ and $\hat{\sigma}_{y}^{2}$ from (6). The estimation procedure is iterative till the convergence is reached (usually in 4-5 steps). After the procedure is finished, computed is the covariance matrix $\boldsymbol{W}$ according to $(7)$.

We have obtained

$$
\left(\begin{array}{l}
\hat{a}-a \\
\hat{b}-b
\end{array}\right) \sim N\left(\left(\begin{array}{l}
0 \\
0
\end{array}\right), \frac{b_{0}^{2} \sigma_{x}^{2}+\sigma_{y}^{2}}{m}\left(\begin{array}{cc}
n & \mathbf{1}^{\prime} \boldsymbol{\mu}_{0} \\
\boldsymbol{\mu}_{0}^{\prime} \mathbf{1} & \boldsymbol{\mu}_{0}^{\prime} \boldsymbol{\mu}_{0}
\end{array}\right)^{-1}\right) .
$$

To emphasize dependence of the distribution on the parameters $\left(\sigma_{x}^{2}, \sigma_{y}^{2}\right)$, we will alternatively denote the covariance matrix of the distribution also by $\boldsymbol{\Phi}\left(\sigma_{x}^{2}, \sigma_{y}^{2}\right)$. If the parameters $\sigma_{x}^{2}, \sigma_{y}^{2}$ are known, $(1-\alpha)$-confidence region for parameters $a, b$ is

$$
\mathcal{C}_{(1-\alpha)}^{*}=\left\{\left(\begin{array}{l}
a \\
b
\end{array}\right): \frac{m}{b_{0}^{2} \sigma_{x}^{2}+\sigma_{y}^{2}} Q^{(E I V)} \leqq \chi_{2}^{2}(1-\alpha)\right\},
$$

where

$$
Q^{(E I V)}=\left(\begin{array}{c}
\hat{a}-a \\
\hat{b}-b
\end{array}\right)^{\prime}\left(\begin{array}{cc}
n & \mathbf{1}^{\prime} \boldsymbol{\mu}_{0} \\
\boldsymbol{\mu}_{0}^{\prime} \mathbf{1} & \boldsymbol{\mu}_{0}^{\prime} \boldsymbol{\mu}_{0}
\end{array}\right)\left(\begin{array}{c}
\hat{a}-a \\
\hat{b}-b
\end{array}\right) .
$$

If $\sigma_{x}^{2}, \sigma_{y}^{2}$ are unknown, we apply the procedure suggested by Kenward and Roger, see [1], to obtain the adjusted Wald-type statistic and its approximate $F$-distribution. This procedure was suggested for small range of measured data (in our case small $m, n$ ). Kenward and Roger proposed a modified estimator of the matrix $\boldsymbol{\Phi}$ of the form

$$
\hat{\boldsymbol{\Phi}}_{A}=\hat{\boldsymbol{\Phi}}-\sum_{i=1}^{2} \sum_{j=1}^{2} w_{i j} \frac{\partial^{2} \boldsymbol{\Phi}}{\partial \sigma_{i}^{2} \partial \sigma_{j}^{2}}
$$


where $\hat{\boldsymbol{\Phi}}=\boldsymbol{\Phi}\left(\hat{\sigma}_{x}^{2}, \hat{\sigma}_{y}^{2}\right), w_{i j}=\{\mathbf{W}\}_{i j}(\mathbf{W}$ is given in $(7))$ and $\sigma_{1}^{2}=\sigma_{x}^{2}, \sigma_{2}^{2}=\sigma_{y}^{2}$. After computations we obtain

$$
\sum_{i=1}^{2} \sum_{j=1}^{2} w_{i j} \frac{\partial^{2} \boldsymbol{\Phi}}{\partial \sigma_{i}^{2} \partial \sigma_{j}^{2}}=0,
$$

so $\hat{\boldsymbol{\Phi}}_{A}=\hat{\boldsymbol{\Phi}}$. The modified estimator $\hat{\boldsymbol{\Phi}}_{A}$ is recommended to use in the statistics

$$
F=\frac{1}{2}\left(\begin{array}{c}
\hat{a}-a \\
\hat{b}-b
\end{array}\right)^{\prime} \hat{\mathbf{\Phi}}_{A}^{-1}\left(\begin{array}{c}
\hat{a}-a \\
\hat{b}-b
\end{array}\right) .
$$

Further approximation of $F$ is in such a way that $\lambda F$ is $F_{2, u}$ distributed (Fisher-Snedecor distribution with 2 and $u$ degrees of freedom). Analogically as Kenward and Roger considerations after tedious computations we obtain

$$
\lambda=1
$$

and

$$
u=(m n-2)+\frac{2 b_{0}^{2} \hat{\sigma}_{x}^{2} \hat{\sigma}_{y}^{2}(m-1) n}{b_{0}^{4} \hat{\sigma}_{x}^{4}+\hat{\sigma}_{y}^{4}} .
$$

If the true values of calibration line coefficients are $a$ and $b$, then the following (approximative) distribution is valid:

$$
\begin{aligned}
F= & \frac{1}{2} \frac{m}{b_{0}^{2} \hat{\sigma}_{x}^{2}+\hat{\sigma}_{y}^{2}}\left(\begin{array}{c}
\hat{a}-a \\
\hat{b}-b
\end{array}\right)^{\prime}\left(\begin{array}{cc}
n & \mathbf{1}^{\prime} \boldsymbol{\mu}_{0} \\
\boldsymbol{\mu}_{0}^{\prime} \mathbf{1} & \boldsymbol{\mu}_{0}^{\prime} \boldsymbol{\mu}_{0}
\end{array}\right) \\
& \times\left(\begin{array}{c}
\hat{a}-a \\
\hat{b}-b
\end{array}\right) \stackrel{\text { approx. }}{\sim} F_{2, u} .
\end{aligned}
$$

From that we get

$$
\begin{aligned}
\operatorname{Pr}\left\{\frac{1}{2} \frac{m}{b_{0}^{2} \hat{\sigma}_{x}^{2}+\hat{\sigma}_{y}^{2}}\left(\begin{array}{c}
\hat{a}-a \\
\hat{b}-b
\end{array}\right)^{\prime}\left(\begin{array}{cc}
n & \mathbf{1}^{\prime} \boldsymbol{\mu}_{0} \\
\boldsymbol{\mu}_{0}^{\prime} \mathbf{1} & \boldsymbol{\mu}_{0}^{\prime} \boldsymbol{\mu}_{0}
\end{array}\right)\right. \\
\left.\quad \times\left(\begin{array}{c}
\hat{a}-a \\
\hat{b}-b
\end{array}\right) \leq F_{2, u}(1-\alpha)\right\} \stackrel{\text { approx. }}{=} 1-\alpha,
\end{aligned}
$$

$\left(F_{t, u}(1-\alpha)\right.$ is the $(1-\alpha)$-quantile of $F_{t, u}$ distribution). So,

$$
\mathcal{C}_{(1-\alpha)}^{(E I V)}=\left\{\left(\begin{array}{l}
a \\
b
\end{array}\right): \frac{m}{2\left(b_{0}^{2} \hat{\sigma}_{x}^{2}+\hat{\sigma}_{y}^{2}\right)} Q^{(E I V)} \leqq F_{2, u}(1-\alpha)\right\}
$$

is the EIV (approximative) $(1-\alpha)$-confidence region for $(a, b)^{\prime}$.

\section{Results}

\subsection{Scheffé-type confidence region for the calibration line}

By applying the Scheffé's method, see [7], we directly get the $100 \times(1-\alpha) \%$-confidence region for the calibration line $a+b \mu$ for all $\mu$. In the case of the maximum likelihood method we get

$$
\begin{aligned}
& \operatorname{Pr}\{|(\tilde{a}+\tilde{b} \mu)-(a+b \mu)| \\
\leq & \left.\sqrt{\chi_{2}^{2}(1-\alpha) \frac{\tilde{b}^{2} \tilde{\sigma}_{x}^{2}+\tilde{\sigma}_{y}^{2}}{m}\left(\frac{1}{n}+\frac{(\mu-\tilde{\tilde{\mu}})^{2}}{\tilde{\boldsymbol{\mu}}^{\prime} \tilde{\boldsymbol{\mu}}-n \tilde{\tilde{\mu}}^{2}}\right)}\right\}=1-\alpha,
\end{aligned}
$$

where $\tilde{\boldsymbol{\mu}}=\left(\tilde{\mu}_{1}, \ldots, \tilde{\mu}_{n}\right)$ and $\overline{\tilde{\mu}}=\left(\mathbf{1}^{\prime} \tilde{\boldsymbol{\mu}}\right) / n$.

In the case of using the errors-in-variables model and the Kenward-Roger approximation we get

$$
\begin{aligned}
& \operatorname{Pr}\{|(\hat{a}+\hat{b} \mu)-(a+b \mu)| \\
\leq & \left.\sqrt{2 F_{2, u}(1-\alpha) \frac{b_{0}^{2} \hat{\sigma}_{x}^{2}+\hat{\sigma}_{y}^{2}}{m}\left(\frac{1}{n}+\frac{\left(\mu-\bar{\mu}_{0}\right)^{2}}{\boldsymbol{\mu}_{0}^{\prime} \boldsymbol{\mu}_{0}-n \bar{\mu}_{0}^{2}}\right)}\right\}=1-\alpha,
\end{aligned}
$$

where $\bar{\mu}_{0}=\left(\mathbf{1}^{\prime} \boldsymbol{\mu}_{0}\right) / n$.

This is directly used for the multiple-use linear univariate calibration, i.e. for measuring with calibrated device.

\subsection{Multiple-use calibration - measuring with calibrated device}

We will assume that the future measurement realized by the calibrated (less precise) measurement device A, say $x$, is a realization of a random variable $X$, distributed as $X \sim N\left(\mu_{x}, \sigma_{x}^{2}\right)$, where $\mu_{x}$ represents the unobservable true value of the measurand.

First, we suggest to construct the approximate $(1-\alpha)$ confidence region for the calibration line, for small significance level $\alpha \in(0,1)$, chosen by the user, according to $(8)$ or $(9)$.

Second, for small significance level $\gamma \in(0,1)$, we suggest to construct the approximate $(1-\gamma)$-confidence interval for $\mu_{x}$. For that we suggest to construct $t$-statistic with approximate $t_{v}$ Student's $t$ distribution

$$
t=\frac{X-\mu_{x}}{\tilde{\sigma}_{x}} \stackrel{\text { approx }}{\sim} t_{\tilde{v}}, \quad \text { resp. } \quad t=\frac{X-\mu_{x}}{\hat{\sigma}_{x}} \stackrel{\text { approx }}{\sim} t_{\hat{v}},
$$

where the degrees of freedom are approximated by the Satterthwaite's approximation, see [8]. In the case of ML method

$$
\tilde{v}=n m,
$$

see [6]. In the case of EIV approach

$$
\hat{v}=\frac{2 \hat{\sigma}_{x}^{4}}{w_{11}},
$$

where $w_{11}$ is element of the matrix $\boldsymbol{W}$ given in (7). 
This leads to the approximate $(1-\gamma)$-confidence interval for unobservable value $\mu_{x}$ :

$\mu_{x} \in\left\langle x-\tilde{\sigma}_{x} t_{\tilde{v}}(1-\gamma / 2), x+\tilde{\sigma}_{x} t_{\tilde{v}}(1-\gamma / 2)\right\rangle=\left\langle\tilde{\mu}_{x l}, \tilde{\mu}_{x u}\right\rangle$

resp.

$\mu_{x} \in\left\langle x-\hat{\sigma}_{x} t_{\hat{v}}(1-\gamma / 2), x+\hat{\sigma}_{x} t_{\hat{v}}(1-\gamma / 2)\right\rangle=\left\langle\hat{\mu}_{x l}, \hat{\mu}_{x u}\right\rangle$, $t_{v}(1-\gamma / 2)$ is the $(1-\gamma / 2)$-quantile of Student's $t$ distribution with $v$ degrees of freedom.

The suggested interval estimator for $\nu_{x}$ is given as the intersection of the bounds of the Scheffé-type $(1-\alpha)$ confidence region for the whole calibration line $a+b \mu$ and the limits of the $(1-\gamma)$-confidence interval for $\mu_{x}$, and is given as $\left\langle\tilde{\nu}_{x l}, \tilde{\nu}_{x u}\right\rangle$ (ML method) or $\left\langle\hat{\nu}_{x l}, \hat{\nu}_{x u}\right\rangle$ (EIV method). In fact,

$$
\begin{aligned}
\tilde{\nu}_{x l}= & \tilde{a}+\tilde{b} \tilde{\mu}_{x l} \\
& -\sqrt{\chi_{2}^{2}(1-\alpha) \frac{\tilde{b}^{2} \tilde{\sigma}_{x}^{2}+\tilde{\sigma}_{y}^{2}}{m}\left(\frac{1}{n}+\frac{\left(\tilde{\mu}_{x l}-\overline{\tilde{\mu}}\right)^{2}}{\tilde{\boldsymbol{\mu}}^{\prime} \tilde{\boldsymbol{\mu}}-n \overline{\tilde{\mu}}^{2}}\right)} \\
\tilde{\nu}_{x u}= & \tilde{a}+\tilde{b}_{\tilde{\mu}_{x u}} \\
& +\sqrt{\chi_{2}^{2}(1-\alpha) \frac{\tilde{b}^{2} \tilde{\sigma}_{x}^{2}+\tilde{\sigma}_{y}^{2}}{m}\left(\frac{1}{n}+\frac{\left(\tilde{\mu}_{x u}-\overline{\tilde{\mu}}\right)^{2}}{\tilde{\boldsymbol{\mu}}^{\prime} \tilde{\boldsymbol{\mu}}-n \overline{\tilde{\mu}}^{2}}\right)},
\end{aligned}
$$

for the ML method and

$$
\begin{aligned}
\hat{\nu}_{x l}= & \hat{a}+\hat{b} \hat{\mu}_{x l} \\
& -\sqrt{2 F_{2, u}(1-\alpha) \frac{\hat{b}^{2} \hat{\sigma}_{x}^{2}+\hat{\sigma}_{y}^{2}}{m}\left(\frac{1}{n}+\frac{\left(\hat{\mu}_{x l}-\bar{\mu}_{0}\right)^{2}}{\boldsymbol{\mu}_{0}^{\prime} \boldsymbol{\mu}_{0}-n \bar{\mu}_{0}^{2}}\right)} \\
\hat{\nu}_{x u}= & \hat{a}+\hat{b} \hat{\mu}_{x u} \\
& +\sqrt{2 F_{2, u}(1-\alpha) \frac{\hat{b}^{2} \hat{\sigma}_{x}^{2}+\hat{\sigma}_{y}^{2}}{m}\left(\frac{1}{n}+\frac{\left(\hat{\mu}_{x u}-\bar{\mu}_{0}\right)^{2}}{\boldsymbol{\mu}_{0}^{\prime} \boldsymbol{\mu}_{0}-n \bar{\mu}_{0}^{2}}\right)},(11)
\end{aligned}
$$

for the EIV based approach. Using Bonferroni's inequality, the intervals (10) and (11) are (approximative) at least $(1-\alpha-\gamma)$-confidence intervals for the (unobservable) value $\nu_{x}$. Preliminary simulation study indicated that the suggested confidence intervals are conservative, i.e. "safe" and appropriate for metrological applications.

\subsection{Example}

In order to illustrate numerically the suggested methods for multiple-use linear calibration case, we have generated a set of artificial calibration data - a possible outcome of simple linear calibration experiment with replicated measurements, see Table 1.

Based on the calibration data from the considered experiment we get the estimated values of the model parameters:

- Based on the MLE method we get $\tilde{a}=0.7405, \tilde{b}=$ $1.4522, \tilde{\sigma}_{x}^{2}=0.1091, \tilde{\sigma}_{y}^{2}=0.0038, \tilde{\mu}_{1}=0.8938$, $\tilde{\mu}_{2}=2.9491, \tilde{\mu}_{3}=5.0120, \tilde{\mu}_{4}=7.0903, \tilde{\mu}_{5}=9.1047$, and $\tilde{v}=15$.
Table 1. Example data from comparative linear calibration experiment. The values $x_{i j}, i=1, \ldots n, j=1, \ldots, m$, represent $m$-times $(m=3)$ replicated measurements of $n$ measurands $(n=5)$ with true values $\mu_{i}=\left(\nu_{i}-a\right) / b$ measured in units of the less precise measurement device. The values $y_{i j}$, $i=1, \ldots n, j=1, \ldots, m$, are the measurements of the measurands with their true values $\nu_{i}=a+b \mu_{i}$, measured in units of the more precise measurement device. Here, the true (unobservable) values of the model parameters are $a=0.5, b=1.5$, $\mu_{1}=1, \mu_{2}=3, \mu_{3}=5, \mu_{4}=7$, and $\mu_{5}=9$, and $\nu_{1}=2$, $\nu_{2}=5, \nu_{3}=8, \nu_{4}=11$, and $\nu_{5}=14$. The observed values $x_{i j}$ and $y_{i j}$ are realizations of mutually independent random variables $X_{i j} \sim N\left(\mu_{i}, \sigma_{x}^{2}\right)$ and $Y_{i j} \sim N\left(\nu_{i}, \sigma_{y}^{2}\right)$, respectively, with the true variances $\sigma_{y}^{2}=0.15$ and $\sigma_{y}^{2}=0.01$.

\begin{tabular}{ccccccccc}
\hline$i$ & $\mu_{i}$ & $x_{i 1}$ & $x_{i 2}$ & $x_{i 3}$ & $\nu_{i}$ & $y_{i 1}$ & $y_{i 2}$ & $y_{i 3}$ \\
\hline 1 & 1 & 0.6086 & 0.7507 & 1.0000 & 2 & 2.0896 & 2.0569 & 1.9766 \\
2 & 3 & 3.2380 & 3.1473 & 2.8769 & 5 & 5.0731 & 4.9744 & 5.0118 \\
3 & 5 & 5.1966 & 4.6092 & 5.4241 & 8 & 8.0578 & 7.9623 & 8.0315 \\
4 & 7 & 7.6555 & 6.9924 & 6.2742 & 11 & 11.0040 & 10.9704 & 11.1444 \\
5 & 9 & 9.2290 & 8.9813 & 9.1658 & 14 & 14.0677 & 13.8525 & 13.9649 \\
\hline
\end{tabular}

For chosen $\alpha=0.01$, the $(1-\alpha)$-quantile of $\chi^{2}$ distribution with 2 degrees of freedom is $\chi_{2}^{2}(1-\alpha)=$ 9.2103. For chosen $\gamma=0.05$, the $(1-\gamma / 2)$-quantile of $t$-distribution with $\tilde{v}$ degrees of freedom is $t_{\tilde{v}}(1-$ $\gamma / 2)=2.1314$

- Based on the EIV approach (linearized EIV model with application of the Kenward-Roger method for estimation of the model parameters) we get $\hat{a}=0.7405$, $\hat{b}=1.4522, \hat{\sigma}_{x}^{2}=0.1264, \hat{\sigma}_{y}^{2}=0.0057, \hat{\mu}_{1}=0.8933$, $\hat{\mu}_{2}=2.9497, \hat{\mu}_{3}=5.0123, \hat{\mu}_{4}=7.0897, \hat{\mu}_{5}=9.1048$, and $u=13.4244$. The element $w_{11}$ of the matrix $\boldsymbol{W}$ is $w_{11}=0.0025$, and from that we get $\hat{v}=12.8762$.

For chosen $\alpha=0.01$, the $(1-\alpha)$-quantile of $F$ distribution with 2 and $u$ degrees of freedom is $F_{2, u}(1-$ $\alpha)=6.6178$. For chosen $\gamma=0.05$, the $(1-\gamma / 2)$ quantile of $t$-distribution with $\hat{v}$ degrees of freedom is $t_{\hat{v}}(1-\gamma / 2)=2.1625$.

After calibration, the less precise device can be used (multiple-times) for estimation of the true value of measurand $\nu_{x}$ together with its (approximate) confidence interval, based on observed value $x$, which is considered to be a realization of random variable $X \sim N\left(\mu_{x}, \sigma_{x}^{2}\right)$.

Consider measurement of the measurand whose true (unobservable) value in units of the more precise device is $\nu_{x}=11.75$. For estimation of the value $\nu_{x}$ we can use only the less precise device (in possibly different units). For example, here we have generated measurement $x=7.1097$ as a realization of $X \sim N\left(\mu_{x}, \sigma_{x}^{2}\right)$ with $\mu_{x}=(11.75-0.5) / 1.5=7.5$ and $\sigma_{x}^{2}=0.15$. For chosen $\alpha=0.01$ and $\gamma=0.05$ we get the following confidence intervals for the true value $\nu_{x}$ :

- Based on the MLE method for estimation of the model parameters from (10) we get $\left\langle\tilde{\nu}_{x l}, \tilde{\nu}_{x u}\right\rangle=\langle 9.6223,12.6138\rangle$ as an approximate, at least $94 \%$, confidence interval for the (unobservable) value $\nu_{x}=0.5+1.5 \times 7.5=11.75$. 


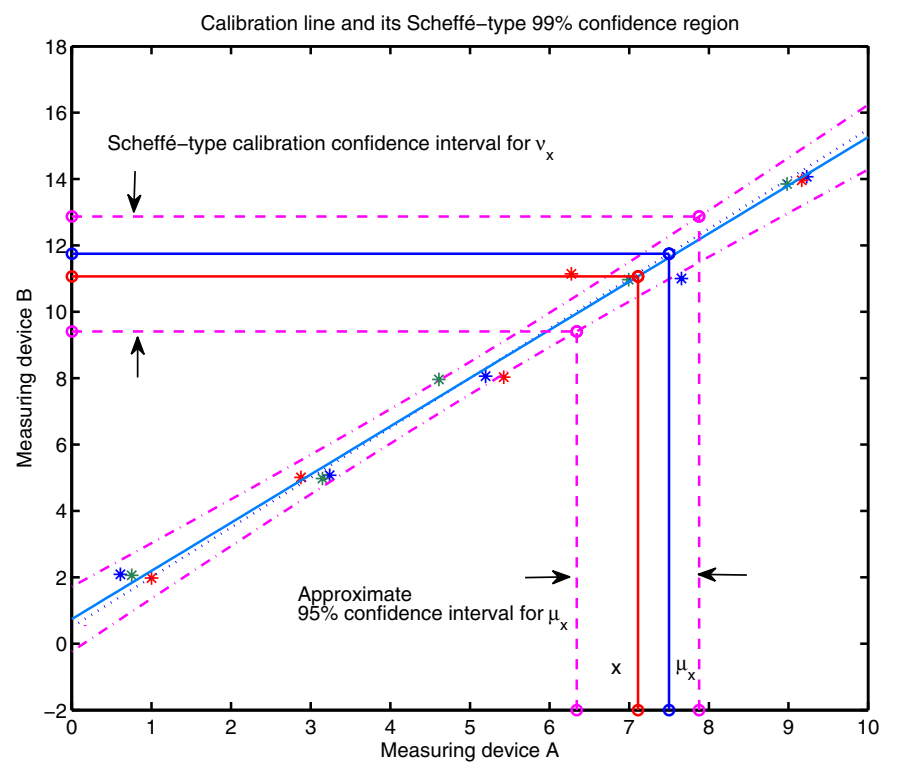

Fig. 1. Calibration line and its Scheffé-type confidence region constructed by the EIV approach. The thick dotted line represents the true calibration line, the solid line is the estimated calibration line together with the limits of the $99 \%$ confidence region (dashed-dotted lines). The dashed lines represent the Scheffé-type interval estimator for $\nu_{x}=a+b \mu_{x}$, where $\mu_{x}=7.5$, based on $x=7.1097$, the realization of random variable $X \sim N(7.5,0.15)$.

- Based on the EIV model and the Kenward-Roger method for estimation of the model parameters from (11) we get $\left\langle\hat{\nu}_{x l}, \hat{\nu}_{x u}\right\rangle=\langle 9.4096,12.8699\rangle$ as an approximate, at least $94 \%$, confidence interval for the (unobservable) value $\nu_{x}=0.5+1.5 \times 7.5=11.75$.

Figure 1 illustrates construction of the approximate $94 \%$ confidence interval for the (unobservable) value $\nu_{x}=11.75$ based on the observed value $x=7.1097$ by the EIV approach. In Figure 2 are plotted the bounds of the approximate MLE and EIV 94\%-confidence regions for $\nu_{x}$, see equations (10) and (11), for arbitrary observation $x$.

\section{Discussion}

As expected, the confidence interval for the true value of measurand, by using the calibrated measuring device and obtained via the EIV method, is in the considered example wider than the confidence interval obtained via the ML method. The EIV method with the Kenward-Roger approximation is suitable for small sample case (as it was suggested), while the ML method is based on asymptotic theory, i.e. is suitable for large samples. But this phenomenon still needs further statistical investigations, as well as the study of the statistical properties of suggested estimators with regard to true values of the calibration model parameters.

Acknowledgements. The work was supported by the Slovak Research and Development Agency, grant APVV-0096-10, and

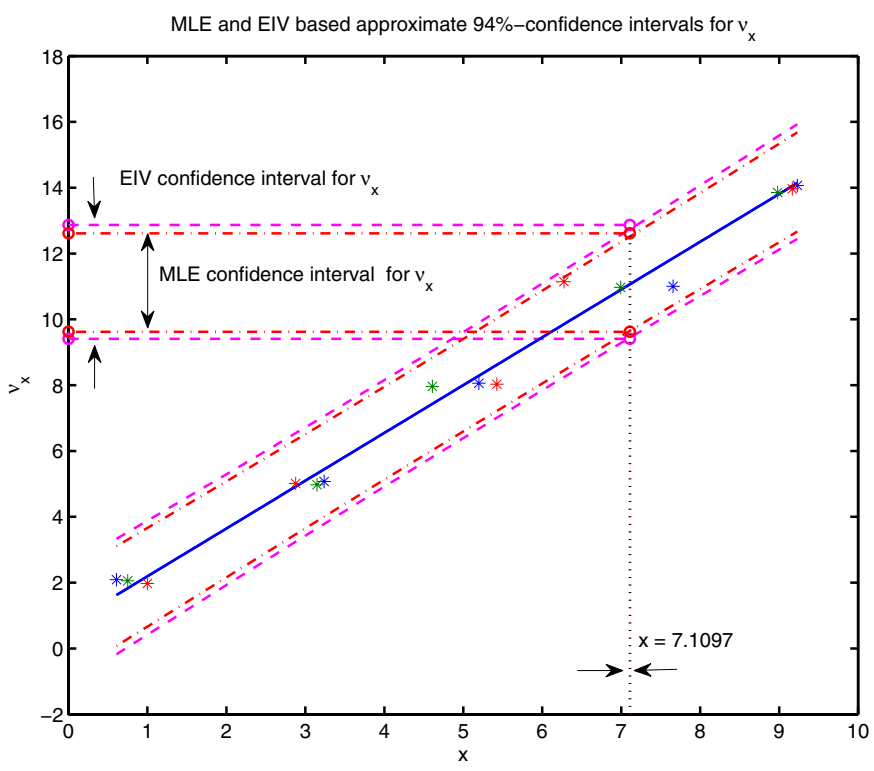

Fig. 2. Estimated calibration line and the approximate MLE and EIV confidence bounds for $\nu_{x}$. The solid line is the estimated calibration line. The dashed-dotted lines represent the interval estimator for $\nu_{x}=a+b \mu_{x}$ based on the MLE method and the dashed lines represent the interval estimator for $\nu_{x}=$ $a+b \mu_{x}$ based on the EIV method. Here $x=7.1097$ is the realization of random variable $X \sim N(7.5,0.15)$. Based on the MLE method, see (10), we get $\left\langle\tilde{\nu}_{x l}, \tilde{\nu}_{x u}\right\rangle=\langle 9.6223,12.6138\rangle$. Based on the EIV model and the Kenward-Roger method, see (11), we get $\left\langle\hat{\nu}_{x l}, \hat{\nu}_{x u}\right\rangle=\langle 9.4096,12.8699\rangle$.

by the Scientific Grant Agency of the Ministry of Education of the Slovak Republic and the Slovak Academy of Sciences, grants VEGA 2/0038/12, 2/0019/10.

\section{References}

1. M.G. Kenward, J.H. Roger, Small sample inference for fixed effects from restricted maximum likelihood, Biometrics 53, 983-997 (1997)

2. G. Wimmer, V. Witkovský, A. Savin, Confidence region for parameters in replicated errors in variables model, in Proceedings in Computational Statistics (COMPSTAT 2004), Prague, Czech Republic (Physica-Verlag, 2004), pp. 1987-1994

3. G. Wimmer, V. Witkovský, Scheffé-type confidence region for the calibration line, Austrian J. Stat. 35, 397-406 (2006)

4. G. Wimmer, V. Witkovský, Linear comparative calibration with correlated measurements, Kybernetika 43, 443-452 (2007)

5. G. Wimmer, V. Witkovský, Univariate linear calibration via replicated errors-in-variables model, J. Stat. Comp. Simul. 77, 213-227 (2007)

6. R. Matušeková, Linear calibration, J. Electr. Eng. 57, 114116 (2006)

7. H. Scheffé, The Analysis of Variance (Wiley, New York, 1959)

8. F.E. Satterthwaite, An approximate distribution of estimates of variance components, Biomet. Bull. 2, 110-114 (1946) 